\title{
OPTIMAL LATENT PERIOD IN A BACTERIOPHAGE POPULATION MODEL STRUCTURED BY INFECTION-AGE
}

\author{
ÀNGEL CALSINA, JOSEP M. PALMADA, AND JORDI RIPOLL
}

\begin{abstract}
We study the lysis timing of a bacteriophage population by means of a continuously infection-age-structured population dynamics model. The features of the model are the infection process of bacteria, the natural death process, and the lysis process which means the replication of bacteriophage viruses inside bacteria and the destruction of them. We consider that the length of the lysis timing (or latent period) is distributed according to a general probability distribution function. We have carried out an optimization procedure and we have found the latent period corresponding to the maximal fitness (i.e. maximal growth rate) of the bacteriophage population.
\end{abstract}

\section{INTRODUCTION}

Most Bacteriophage viruses (etymologically "bacteria eater") replicate inside bacteria causing the death of their host. This process starts when a phage (for short) is adsorbed by the receptors of the cell membrane and injects its genetical material through it. After some time interval the cell machinery of the bacterium synthesizes copies of the virus nucleic acid, the proteins of the capsules and the tails of the new phages. Finally the bacterium lyses ("explodes" and dies) releasing an amount of new virions (called burst size) which widely varies between 5 and 250 depending on the strain. This process, replication of phages linked to destruction of bacteria, obviously indicates that the treatment of bacterial infections using phages can be useful as a therapeutic tool. Indeed, after being almost forgotten due to the discovery of antibiotics, the so called phage therapy is nowadays becoming more popular since the emergence of the antibiotic resistances. In particular, this work, though it has no therapeutic implications, was partially motivated by a research project of the Department of Genetics and Microbiology at the Universitat Autònoma de Barcelona, whose goal is the control of Salmonella infections in animal farms by means of bacteriophages.

The already mentioned period of time between the infection (the adsorption of a virus particle by a bacterium) and the lysis is called lysis timing or latent period. According to experimentalists, the average length of the lysis timing is from 15 minutes (such a short latency time has been observed in the adsorption

Date: October 2009.

Key words and phrases. Phage infection; random lysis timing; partial differential equations; delay equations; fitness optimization. 
of a phage called C78 by a strain of Salmonella enterica) up to 45 minutes in the case of other Salmonella phages for instance. In this paper we focus on this latent period and how is related to the virus population growth rate. More precisely, we compute the latent period giving the maximal growth rate of the phage population (the maximal fitness), among a very general form of latent periods. In [8] a similar computation was performed in order to find the optimal age of sex-reversal in sequential hermaphrodite populations, also assuming an a priori very general form of the possible distributions of age of sex-reversal.

In many papers of epidemiological models, the latent periods (understood in general as the period when infected individuals are not infectious yet) are typically assumed to be either exponentially distributed or fixed. This assumptions yield ordinary differential equations systems in the first case, and delay differential equations in the second one.

In the present paper we introduce and analyze a structured bacteriophage population model where we extend the previous assumption in the sense that we consider a general probability distribution function for the length of the latent period.

In the literature we find several papers on the dynamics of marine plankton bacteriophage infections. In [5] the latent period is assumed to be exponentially distributed and in [6] instead, the latent period has a fixed length. Moreover, in [10] the authors include in addition spatial diffusion of the population.

On the other hand, see $[1,2,18,17]$ for models and experimental results on the latent period from the evolutionary point of view.

The paper is organized as follows. In Section 2 a bacteriophage infection is described and an (infection age)-structured population model with a general lysis timing is introduced. Two versions of the system are stated depending on either the probability distribution of the latent period is assumed to be absolutely continuous (to have a density function) or not. In the first case the model reduces to an age-structured population dynamics system given by a partial differential equation coupled to an integro-differential equation whereas the second, which includes the former as a particular case, is directly formulated as a delay equation. Existence and uniqueness of global solutions, which yield a strongly continuous semigroup, is shown in Appendix A through the reduction to a single integral equation for the number of phages. In Section 3, the bacteriophage fitness is defined as the growth bound of the solution semigroup. Exponentially growing solutions (separate variables solutions) are computed. The control of this growth bound is reduced to the computation of the eigenvalues of the infinitesimal generator. There exists at most one real eigenvalue. Finally, in Section 4 the maximal latent period is computed and corresponds to a fixed length period even if the lysis process may take place at a different infection-age for each bacterium. 


\section{Model FORMULATiON}

We consider a micro-epidemiology model made up by free bacteriophage viruses infecting a population of bacteria with variable lysis timing (also called latent period) as the main new feature of the model.

In the lytic cycle, the interaction between phages and bacteria is described as follows: viruses attack susceptible bacteria which get infected in the sense that a virus successfully injects its genetical material (adsorption) through the bacterial membrane. Then, after an eclipse period $E \geq 0$ (where the bacterium is forced to manufacture viral products which will become part of the new virions), the assembly of new virions starts until the bacterium dies by lysis, that is, the bacterium explodes releasing new virions which are then free to attack other infected-free bacteria. The number of particles released in each lysis is called the burst size. See the diagram in Figure 1 for an schematic representation of the process. Let us remark that we are considering a type of bacteriophages (e.g. T4 phages) that inhibits the replication of the infected bacteria, so only uninfected bacteria are capable of reproducing by division, see [17] p. 22, or [2] p. 4234. We think these populations inhabiting in a solution and measured in units of number of viruses/bacteria per unit of volume.

The variability of the lysis timing in the bacterial population is incorporated into the model by considering that this latent period $T>0$, which is defined as the time elapsed between infection and lysis, is a positive random variable with a given probability distribution function $P(T \leq \tau)=F(\tau)$ where $\tau$ is the time since infection. So, the precise meaning of the latent period in the present paper is the random period between the moment of being infected and the moment of releasing the infecting agent. The eclipse period $E$ (i.e. from infection until the appearance of new virions inside the bacterium) constrains the random latent period in the sense that $P(T \leq E)=0$.

Bacteria are divided according to the disease stage: uninfected (susceptible) $S(t)$ and infected (but not infectious). Since we are considering that the lysis timing (latent period) may differ from one bacterium to another, we introduce the variable $v(\tau, t)$ as the density of infected bacteria with respect to the infection age (i.e. the time that has passed since the infection) at time $t$. On the other hand, the infecting agent is the free bacteriophage virus population $P(t)$.

We also consider the demographic process of natural mortality in both populations: let $\delta, m>0$ be the constant per capita mortality rates for viruses and bacteria respectively.

Finally, let us point out that we are considering populations homogeneously distributed in space, and that super-infections are ignored.

For a general non-linear model, one can consider that the dynamics for the susceptible bacteria in absence of viruses is given by $S^{\prime}(t)=r(S(t), t)$ with the latter function being defined according to a specific situation, whereas in case of viral infection one has $S^{\prime}(t)=r(S(t), t)-k S(t) P(t)$ if we assume that the incidence 
rate (number of new infected bacteria per unit of time) follows the law of mass action. The proportionality factor $k>0$ represents the adsorption rate. However, in the present paper, we will assume that the population of susceptible bacteria is at equilibrium $S(t)=S^{*}$. For simplicity, on the forthcoming we will denote by $S$ the constant $S^{*}$. For instance one can think in a laboratory population where there is a suitable inflow of uninfected bacteria in order to maintain constant this population. Also one can think in the initial phase of the infection where a small amount of phages are introduced in a population of totaly infected-free bacteria at equilibrium and a linear exponential growth/decay of the infected bacteria and phages takes place.

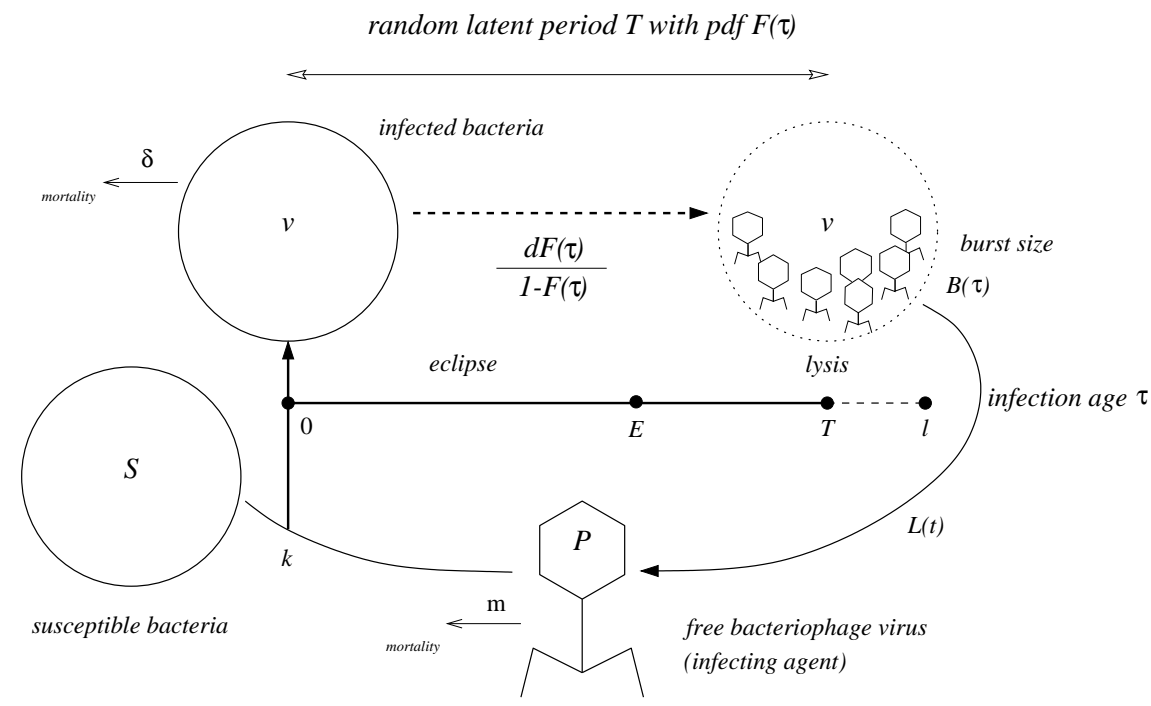

FiguRE 1. Viral-Bacteria model. Free bacteriophages ("bacteriaeater") encounter susceptible bacteria that become infected at a rate $k S P$, where $k$ is the adsorption rate. The latent period $T$, the time-span from infection to lysis which include an eclipse period $E$, is taken as a random variable. After lysis, new virions are released according to $L(t)=\int_{0}^{l} B(\tau) v(\tau, t) \frac{d F(\tau)}{1-F(\tau)}$, where $B(\tau)$ is the burst size and $F(\tau)$ is the probability distribution of the latent period.

Now, let us assume for a while that the latent period $T>0$ is an absolutely continuous random variable, i.e. its probability distribution function is an absolutely continuous function. In this case the (infection age)-structured model can be described by the following linear system which is a combination of a first order partial differential equation with a boundary condition and an ordinary differential equation: 


$$
\left\{\begin{array}{l}
\frac{\partial v}{\partial t}(\tau, t)+\frac{\partial v}{\partial \tau}(\tau, t)=-\delta v(\tau, t)-\frac{F^{\prime}(\tau)}{1-F(\tau)} v(\tau, t), \quad \tau<l, \\
v(0, t)=k S P(t), \\
\frac{d P}{d t}(t)=-m P(t)-k S P(t)+\int_{0}^{l} B(\tau) v(\tau, t) \frac{d F(\tau)}{1-F(\tau)} .
\end{array}\right.
$$

We recall that the latent period is distributed according to $F(\tau)$ (which is nondecreasing and $F(0)=0)$ and, accordingly, the maximum age of infection $l(>E)$ is given by $l:=\sup \{\tau: F(\tau)<1\} \leq \infty$, that is, the lysis may occur between 0 and $l$, and the rate $\frac{F^{\prime}(\tau)}{1-F(\tau)}$ represents the per capita virus-induced mortality rate or the per capita lysis rate. Notice that if the maximum age of infection is finite (the maximum value of the latent period) then it is $l=F^{-1}(1)$, with the latter being the generalized inverse of $F$.

On the other hand, the burst size $B(\tau)$ (the amount of new virions released per lysis) as a function of the infection age at lysis is assumed to be bounded and continuous, $B(\tau) \equiv 0$ for $\tau \leq E$, and strictly increasing with $B^{\prime \prime}(\tau)<0$ for $\tau>E$. Moreover, the maximum burst size $\lim _{\tau \rightarrow l} B(\tau)=R>1$ since otherwise there is no possibility for the spread of the infection. The quantity $R$ is interpreted as a measure of the quality of the bacteria. The total amount of new virions released per unit of time is given by $\int_{0}^{l} B(\tau) v(\tau, t) \frac{d F(\tau)}{1-F(\tau)}=\int_{0}^{l} B(\tau) v(\tau, t) \frac{F^{\prime}(\tau)}{1-F(\tau)} d \tau$.

The lysis rate and the number of particles released are derived as follows. The number of lysis occurred in the time interval from $t$ to $t+d t$ of bacteria with infection age between $\tau<l$ and $\tau+d \tau$ is the number of bacteria with infection age between $\tau$ and $\tau+d \tau$, at time $t$, times the probability that a $\tau$-aged bacterium at time $t$ dies by lysis between $t$ and $t+d t$. In symbols, $v(\tau, t) d \tau P(\tau<$ $T \leq \tau+d t \mid T>\tau)=v(\tau, t) d \tau \frac{P(\tau<T \leq \tau+d t)}{P(T>\tau)}=v(\tau, t) d \tau \frac{F(\tau+d t)-F(\tau)}{1-F(\tau)}$. Therefore, dividing by $d t$ and taking the limit as $d t \rightarrow 0$, one has that the measure of lysis per unit of time is $v(\tau, t) \frac{F^{\prime}(\tau)}{1-F(\tau)} d \tau=v(\tau, t) \frac{d F(\tau)}{1-F(\tau)}$, that is, the instantaneous lysis rate at infection age $\tau$ is $v(\tau, t) \frac{F^{\prime}(\tau)}{1-F(\tau)}$. Finally, multiplying the measure of lysis by the burst size and integrating over the age-span, one gets the total number of new virions released per unit of time $\int_{0}^{l} B(\tau) v(\tau, t) \frac{d F(\tau)}{1-F(\tau)}$. Let us point out that this total number, for some initial condition $(v(\tau, 0), P(0))=\left(v_{0}(\tau), P_{0}\right)$, with $v_{0}(\cdot) \in L_{+}^{1}(0, l)$ and $P_{0} \geq 0$, and for a particular probability distribution, could be infinite.

In order to extend the model to a general random variable $T>0$, e.g. not necessarily absolutely continuous, we can write an 'integrated' version of system (1) where the derivative $F^{\prime}$ of the probability distribution of the latent period disappears from the system. Indeed, defining $\nu:=m+k S>0$, integrating along the characteristic lines the partial differential equation in (1), and using the variation of the constants formula to the ordinary differential equation in (1), 
we get the following general (linear) system

$$
\left\{\begin{array}{l}
v(\tau, t)= \begin{cases}k S P(t-\tau)(1-F(\tau)) e^{-\delta \tau}, & \tau<t \\
v_{0}(\tau-t) \frac{1-F(\tau)}{1-F(\tau-t)} e^{-\delta t}, & \tau>t\end{cases} \\
P(t)=P_{0} e^{-\nu t}+\int_{0}^{t} L(s) e^{-\nu(t-s)} d s, \quad L(t)=\int_{(0, l]} B(\tau) v(\tau, t) \frac{d F(\tau)}{1-F(\tau)},
\end{array}\right.
$$

where $d F$ is defined by $P\left(\tau_{1}<T \leq \tau_{2}\right)=\int_{\left(\tau_{1}, \tau_{2}\right]} d F(\tau)$ as it is usual in probability/measure theory. The system above is interpreted as follows. The first equation says that the density of bacteria with infection age $\tau<t$ at time $t$ is equal to the density of bacteria infected at time $t-\tau, v(0, t-\tau)=k S P(t-\tau)$, times the probability of not yet lysed at age $\tau$ and times the probability of surviving to age $\tau$. On the other hand, the second equation says that the density of bacteria with infection age $\tau>t$ at time $t$ is equal to the initial density of bacteria with infection age $\tau-t, v(\tau-t, 0)=v_{0}(\tau-t)$, times the probability of not yet lysed at age $\tau$ provided that it has no lysed at age $\tau-t$, and times the probability of surviving from age $\tau-t$ to age $\tau$. The third equation in (2) is just the integral version of the linear inhomogeneous ordinary differential equation in (1).

If the latent period $T>0$ is an absolutely continuous random variable, it can be shown that systems (1) and (2) are equivalent in the sense that all solution of (1) with initial condition $\left(v_{0}(\cdot), P_{0}\right)$ is also solution of $(2)$, and that all solution of (2) fulfills the differential system (1) taking into account that the left hand side of the partial differential equation is understood as the 'directional derivative' in the direction of the vector $(1,1)$, and understanding that a solution of $(1)_{3}$ is given by the variation of the constants formula by definition.

In Appendix A, we show the existence and uniqueness of global solutions to the general model (2) which are non-negative and yield a strongly continuous semigroup of bounded (linear) operators. The key point is that system (2) can be rewritten as a single integral equation for the number of phages $P(t)$ :

$$
\begin{aligned}
P(t)=P_{0} e^{-\nu t}+\int_{0}^{t}\left(k S \int_{(0, \bar{s}]} B(\tau) P(s-\tau) e^{-\delta \tau} d F(\tau)+\right. \\
\left.+e^{-\delta s} \int_{(\bar{s}, l]} B(\tau) v_{0}(\tau-s) \frac{d F(\tau)}{1-F(\tau-s)}\right) e^{-\nu(t-s)} d s,
\end{aligned}
$$

with $\bar{s}:=\min \{s, l\}$. Once we know the solution of (3), the density of infected bacteria $v(\tau, t)$ is recovered by the first equation in $(2)$.

Next, let us illustrate two examples which are particular cases of the present model.

Firstly, if we assume a latent period exponentially distributed thereafter the eclipse period, that is, $F(\tau)=1-e^{-\alpha(\tau-E)}$ for $\tau \geq E, \alpha>0$, then the expected latent period turns out to be $E[T]=E+\frac{1}{\alpha}$. If in addition we replace the burst 
size by its mean value $\bar{B}(>1)$ and introduce two new state variables $I_{0}(t):=$ $\int_{0}^{E} v(\tau, t) d \tau$ and $I_{E}(t):=\int_{E}^{\infty} v(\tau, t) d \tau$, then the linear system (1) reduces to the following system of linear delay differential equations

$$
\left\{\begin{array}{l}
\frac{d I_{0}}{d t}(t)=k S P(t)-v(E, t)-\delta I_{0}(t), \\
\frac{d I_{E}}{d t}(t)=v(E, t)-(\delta+\alpha) I_{E}(t), \\
\frac{d P}{d t}(t)=-\nu P(t)+\alpha \bar{B} I_{E}(t),
\end{array}\right.
$$

with $v(E, t):=k S P(t-E) e^{-\delta E}$ for $E<t$, and $v(E, t):=v_{0}(E-t) e^{-\delta t}$ for $E>t$. We recall that $\nu:=m+k S$ and notice that if the eclipse period is neglected, i.e. $E=0$, then the system further reduces to a planar system of ordinary differential equations for the population sizes of infected bacteria and phages. In [5] an ode based ecological model of marine bacteriophages is studied, where the latent period is taken exponentially distributed.

The second example is the following. If we assume a fixed latent period instead, that is, $F(\tau)=\mathcal{X}_{[l, \infty)}(\tau)$ with $E[T]=l>E$, and introduce a new state variable $I(t):=\int_{0}^{l} v(\tau, t) d \tau$ which is the total number of infected bacteria, then from (3) and $(2)$, the model reduces to the following system of linear delay differential equations

$$
\left\{\begin{array}{l}
\frac{d I}{d t}(t)=k S P(t)-v_{-}(l, t)-\delta I(t), \\
\frac{d P}{d t}(t)=-\nu P(t)+B(l) v_{-}(l, t)
\end{array}\right.
$$

with $v_{-}(l, t):=k S P(t-l) e^{-\delta l}$ for $l<t$, and $v_{-}(l, t):=v_{0}(l-t) e^{-\delta t}$ for $l>t$. Indeed, the second equation in (5), which is uncoupled from the first one, is the differential version of equation (3) at $F=\mathcal{X}_{[l, \infty)}$, and the first equation in (5) is the differential form of $I(t)=k S \int_{0}^{\bar{t}} P(t-\tau) e^{-\delta \tau} d \tau+e^{-\delta t} \int_{\bar{t}}^{l} v_{0}(\tau-t) d \tau$, with $\bar{t}:=\min \{t, l\}$, which comes from the first equation in (2) also at $F=\mathcal{X}_{[l, \infty)}$. See [6] and [10] where the previously cited model is treated assuming a fixed value of the latent period which leads to delay equations as in (5). Even though in [10] the authors already suggest the possibility of considering the latent period as given by a probability distribution function, as in systems (1) and (2).

\section{BACTERIOPhaGe FitNess}

3.1. Growth bound of the solution semigroup. From the evolutionary point of view, we are interested in the optimal probability distribution function of the latent period, in the sense that this probability distribution gives the maximal growth rate of the viral population (i.e. the maximal growth bound of the solution semigroup of (2)). 
In order to reduce the control on the growth bound of the semigroup, denoted by $\mathcal{S}(t)$, to the computation of the eigenvalues of its infinitesimal generator, it is very useful the definition of the so-called essential growth bound:

$$
\omega_{e}=\lim _{t \rightarrow 0^{+}} \frac{\ln (d(\mathcal{S}(t), \mathcal{K}))}{t},
$$

where $\mathcal{K}$ is the set of all compact linear operators and, for a bounded linear operator $\mathcal{B}$, the distance to this set is defined by $d(\mathcal{B}, \mathcal{K})=\inf _{K \in \mathcal{K}}\|\mathcal{B}-K\|$. Indeed, the following holds:

$$
\omega_{0}=\max \left\{\omega_{e}, s(A)\right\},
$$

where $s(A)$ is the spectral bound, i.e. the supremum of the real parts of the spectral values of the infinitesimal generator $A$. Moreover, any spectral value with real part larger than $\omega_{e}$ is necessarily an eigenvalue (see for instance [9], Prop. 8.6). Next we show

Proposition 3.1. The essential growth bound of the solution semigroup $\mathcal{S}(t)$ of system (2) is less than or equal to $-\delta$ (the mortality of infected bacteria).

Proof. As usual in structured population dynamics, this follows from a decomposition of the solution semigroup in the form

$$
\mathcal{S}(t)\left(\begin{array}{c}
v_{0}(\cdot) \\
P_{0}
\end{array}\right):=\left(\begin{array}{c}
v(\cdot, t) \mathcal{X}_{[0, \min \{t, l\})}(\cdot) \\
P(t)
\end{array}\right)+\left(\begin{array}{c}
v(\cdot, t) \mathcal{X}_{[\min \{t, l\}, l]}(\cdot) \\
0
\end{array}\right)
$$

where $v(\tau, t)$ is given by (2). The second term on the right hand side is obviously exponentially decaying at a rate at least equal to $-\delta$. The first one is, for a fixed $t$, a compact operator in $X:=L^{1}(0, l) \times \mathbb{R}$ since it can be written as a composition of three bounded linear operators, the second of which is compact. Namely, $\mathcal{T}_{1}$ mapping the initial condition $\left(v_{0}, P_{0}\right)$ in $X$ to the function $P$ in $W^{1,1}(0, t)$ (see Appendix A), $\mathcal{T}_{2}$ mapping $W^{1,1}(0, t)$ to $L^{1}(0, t) \times \mathbb{R}$ and defined by $\mathcal{T}_{2} P=\left(\begin{array}{c}P \\ P(t)\end{array}\right)$, where the first component is the compact Sobolev embedding and the second is the evaluation at the final point of the interval (continuous, and obviously compact, in $\left.W^{1,1}(0, t)\right)$ and, finally, $\mathcal{T}_{3}$ mapping $L^{1}(0, t) \times \mathbb{R}$ in $L^{1}(0, l) \times \mathbb{R}$, its first component given by the first equation in $(2)$ extended to $(0, l)$ as in $(6)$, and the second one by the identity operator in $\mathbb{R}$.

As a consequence, the existence of an eigenvalue with a real part larger than $-\delta$ will imply $\omega_{0}=s(A)$, which is a (real) eigenvalue (the spectral bound of a positive semigroup always belongs to the spectrum of the generator), and hence, it will reduce the computation of $\omega_{0}$ to finding the maximal real solution of the characteristic equation (see below) whenever we show that there is a real solution larger than $\omega_{e}$ of this equation. Actually we will show that the characteristic equation has at most a real solution. 
3.2. Characteristic equation. In this section we are going to compute the point spectrum of the infinitesimal generator of the solution semigroup, in order to determine the growth bound of the semigroup.

Let $A$ be the infinitesimal generator of the solution semigroup $\mathcal{S}(t)$. If $\lambda$ is an eigenvalue of $A$ then $e^{\lambda t}$ is an eigenvalue of the operator $\mathcal{S}(t)$. So, the eigenfunctions, $c(\varphi(\tau), 1)$ with $c$ an arbitrary constant, corresponding to an eigenvalue $\lambda$ are computed as solutions of system (2) in the form:

$$
\left\{\begin{array}{l}
v(\tau, t)=e^{\lambda t} \varphi(\tau) \\
P(t)=e^{\lambda t}
\end{array}\right.
$$

Therefore,

$$
\left\{\begin{array}{l}
e^{\lambda t} \varphi(\tau)= \begin{cases}k S e^{\lambda(t-\tau)}(1-F(\tau)) e^{-\delta \tau} & \tau<t \\
\varphi(\tau-t) \frac{1-F(\tau)}{1-F(\tau-t)} e^{-\delta t} & \tau>t\end{cases} \\
\lambda=-\nu+\widehat{L}, \quad \widehat{L}=\int_{(0, l]} B(\tau) \frac{\varphi(\tau)}{1-F(\tau)} d F(\tau),
\end{array}\right.
$$

where the first equality follows by direct substitution whereas the second one comes from an explicit integration in $(2)_{2}$ after substitution of (7). For fixed $\tau$ and $t>\tau$, the first equation in (8) yields $\varphi(\tau)=k S e^{-(\lambda+\delta) \tau}(1-F(\tau))$, which solves the second part for $\tau>t$, as it is easily checked.

Accordingly, $\widehat{L}=k S \int_{(0, l]} B(\tau) e^{-(\lambda+\delta) \tau} d F(\tau)$ and we get the following characteristic equation for $\lambda \in \mathbb{C}$ :

$$
\lambda=-\nu+k S \int_{(0, l]} B(\tau) e^{-(\lambda+\delta) \tau} d F(\tau) .
$$

Notice that this equation can be written as

$$
1=\frac{k S \int_{(0, l]} B(\tau) e^{-(\lambda+\delta) \tau} d F(\tau)}{\lambda+m+k S},
$$

where the expression in the right hand side at $\lambda=0$ is the parameter $R_{0}$ which is interpreted here as the expected number of virions produced by a phage during its lifetime.

To end up the section, let us show two results about equation (9) which will be used in the next section.

Firstly, let us show that equation (9) implicitly defines a real function $\lambda_{F}$, i.e. a real eigenvalue as a function of the probability distribution $F$. Let us define the function $G$ as

$$
G(\lambda, F):=\lambda+\nu-k S \int_{(0, l]} B(\tau) e^{-(\lambda+\delta) \tau} d F(\tau),
$$

for $\operatorname{Re}(\lambda) \geq-\delta$ and for all probability distribution function $F$. For every probability distribution $F$, if there exists a real value $\lambda_{F}$ such that $G\left(\lambda_{F}, F\right)=0$ then 
it is unique. Indeed, it follows from the fact that the real function $\lambda \in(-\delta, \infty) \mapsto$ $G(\lambda, F)$ is strictly increasing and continuous, and either there exists no zero of the function or there exists only one.

Now, let us see that we can restrict the characteristic equation to real values if we are interested in the rightmost eigenvalue (i.e. the eigenvalue with larger real part).

Lemma 3.1. If $\lambda \in \mathbb{C} \backslash \mathbb{R}$ is such that $G(\lambda, F)=0$, then there exists a unique real value $\tilde{\lambda}$ with $\operatorname{Re}(\lambda)<\tilde{\lambda}$ fulfilling $G(\widetilde{\lambda}, F)=0$.

Proof. First notice $\operatorname{Re} G(\lambda, F) \geq G(\operatorname{Re} \lambda, F)$, and that equality implies

$$
\operatorname{supp} d F \subset\{\tau>0: \cos (\tau \operatorname{Im} \lambda)=1\} \subset\{\tau>0: \sin (\tau \operatorname{Im} \lambda)=0\}
$$

and hence, $\operatorname{Im} G(\lambda, F)=\operatorname{Im} \lambda$. Since $\lambda$ is not real and $G(\lambda, F)=0$, it follows that $G(\operatorname{Re} \lambda, F)<0$. Finally, since for real $z, G(z, F)$ strictly increases and has an infinite limit at infinite, the latter gives the claim.

\section{Optimization of the latent Period}

If condition $m-\delta \geq k S(R-1)$ holds then the growth bound of the solution semigroup $\omega_{0} \leq-\delta<0$. Indeed, let us assume the contrary $\omega_{0}>-\delta$, which implies that the growth bound is equal to the dominant eigenvalue of the infinitesimal generator and therefore it coincides with the unique real solution of the characteristic equation. However, this is not possible since the function $G(\lambda, F)$ is strictly increasing in $\lambda$ and

$$
\begin{aligned}
G(-\delta, F) & =-\delta+\nu-k S \int_{(0, l]} B(\tau) d F(\tau)>-\delta+\nu-k S R \\
& =m-\delta-k S(R-1)>0
\end{aligned}
$$

and therefore the claim follows.

Moreover, since here $\omega_{0}<0$ then it follows that the bacteriophage virus population $P(t)$ goes to extinction. Let us remark that in this case it would make sense to find the probability distribution that optimizes the decay of the population of phages in the sense that the extinction of the phages occurs in the slowest possible way. Nevertheless, we will concentrate on the biologically more interesting case which follows to suppose the strict opposite inequality since then the growth bound of the semigroup can be positive. In this new situation, i.e.

$$
m-\delta<k S(R-1),
$$

we can readily assure that there exists some probability distribution functions $F$ whose real eigenvalue $\lambda_{F}$ exists and $\lambda_{F}>-\delta$. For instance, one can take the distribution $F(\tau):=\mathcal{X}_{\left[\tau_{0}, \infty\right)}(\tau)$ with $\tau_{0}$ any number fulfilling $m-\delta<k S\left(B\left(\tau_{0}\right)-1\right)$. So, we will assume (11) throughout this section.

If $F$ is a probability distribution such that $\lambda_{F}>-\delta$ then, since $\omega_{e} \leq-\delta, \lambda_{F}$ is the growth bound of the solution semigroup and also the dominant eigenvalue 
of the infinitesimal generator (i.e. the unique real solution of the characteristic equation (9)). Next, we will focus on the computation of the probability distribution $\widehat{F}$ which maximizes the non-linear functional $F \mapsto \lambda_{F}$, implicitly defined by $G(\lambda, F)=0$, see $(10)$.

Let $\widetilde{M}$ be the vector space of the real functions $F(x)=\mu([0, x])$ for some real measure $\mu$ on $\mathbb{R}^{+}$such that the norm $\|F\|_{\widetilde{M}}:=\int_{0}^{\infty} e^{-x}|F(x)| d x$ is finite. Notice in particular that these functions are right-continuous. In this space let us define the following subsets:

$$
M:=\{F \in \widetilde{M}: \text { non-decreasing and } F(x) \in[0,1]\}
$$

and $M_{1}$ the subset of $M$ of the functions satisfying in addition that

$$
\lim _{x \rightarrow \infty} F(x)=1 \text {. }
$$

Proposition 4.1. The set $M$ is a compact subset of the normed vector space $\widetilde{M}$.

Proof. Let us consider a sequence $\left\{F_{n}\right\} \in M$. We have to show that there exists a subsequence which is convergent in $M$. By the Helly-Bray Theorem (see e.g. the appendix in [8]), there exists a subsequence $\left\{F_{n_{k}}\right\}$ such that converges pointwise to a non-decreasing and right-continuous function $F$ from $\mathbb{R}^{+}$to $[0,1]$, for all continuity points of the limit function and hence almost everywhere in $\mathbb{R}^{+}$.

On the one hand, $F_{n_{k}}$ tends to $F:|| F_{n_{k}}-F \|_{\widetilde{M}}=\int_{0}^{\infty} e^{-x}\left|F_{n_{k}}(x)-F(x)\right| d x \rightarrow 0$ by the dominated convergence theorem. On the other hand, obviously $F \in M$. Therefore $M$ is a compact set.

Lemma 4.1. Let $\mu$ be a measure on $\mathbb{R}^{+}$and let $\varphi(x)$ be a $\mathcal{C}^{1}$ function, $\mu$-integrable and such that $\lim _{x \rightarrow \infty} \varphi(x)=0$. Then

$$
\int_{[0, \infty)} \varphi(x) d \mu(x)=-\int_{0}^{\infty} \varphi^{\prime}(x) \mu([0, x]) d x .
$$

Proof. By the Theorem of Fubini, one has that

$$
\begin{aligned}
\int_{[0, \infty)} \varphi(x) d \mu(x) & =-\int_{[0, \infty)}\left(\lim _{y \rightarrow+\infty} \varphi(y)-\varphi(x)\right) d \mu(x) \\
& =-\int_{[0, \infty)} \int_{x}^{\infty} \varphi^{\prime}(y) d y d \mu(x) \\
& =-\int_{0}^{\infty} \varphi^{\prime}(y) \int_{[0, y]} d \mu(x) d y \\
& =-\int_{0}^{\infty} \varphi^{\prime}(y) \mu([0, y]) d y
\end{aligned}
$$

Let us rewrite the function $G$ defined in (10) as follows

$$
G(\lambda, F)=\lambda+m+k S(1-L(\lambda) F),
$$


where

$$
L(\lambda) F:=\int_{\mathbb{R}^{+}} B(\tau) e^{-(\lambda+\delta) \tau} d F(\tau)
$$

Proposition 4.2. The following holds:

(1) The functional $L(\lambda): M \rightarrow \mathbb{R}$ is well-defined for all $\lambda \geq-\delta$ and it is continuous if and only if $\lambda>-\delta$.

(2) The function $G:(-\delta, \infty) \times M \rightarrow \mathbb{R}$ is continuous.

Proof. (1) The first part of the claim is obvious. Now, let $\lambda>-\delta$ and let $\left\{F_{n}\right\} \in M$ be a convergent sequence with limit $F \in M$. Let us show $L(\lambda) F_{n} \rightarrow L(\lambda) F$.

First notice that, by Lemma 4.1, $L(\lambda) F_{n}=\int_{0}^{\infty} g(x) F_{n}(x) d x$ and $L(\lambda) F=\int_{0}^{\infty} g(x) F(x) d x$, where $g(x)=\left((\lambda+\delta) B(x)-B^{\prime}(x)\right) e^{-(\lambda+\delta) x}$ is an integrable function since $\lambda>-\delta$. So $L(\lambda) F_{n}$ is a bounded sequence of real numbers. Let us consider any convergent subsequence $L(\lambda) F_{n_{k}}$ and note that, as $F_{n_{k}}$ tends to $F$ in $\widetilde{M}$, i.e., $e^{-x} F_{n_{k}}(x)$ tends to $e^{-x} F(x)$ in $L^{1}$, we then have, for a subsequence, that $e^{-x} F_{n_{k_{l}}}(x) \rightarrow e^{-x} F(x)$ a.e. (see [16]), and consequently, $g(x) F_{n_{k_{l}}}(x) \rightarrow g(x) F(x)$ a.e. . By the dominated convergence theorem,

$$
L(\lambda) F_{n_{k_{l}}}=\int_{0}^{\infty} g(x) F_{n_{k_{l}}}(x) d x \rightarrow \int_{0}^{\infty} g(x) F(x) d x=L(\lambda) F
$$

and hence $L(\lambda) F_{n_{k}} \rightarrow L(\lambda) F$ too. Thus, any convergent subsequence of $L(\lambda) F_{n}$ has the same limit and the claim follows.

On the other hand, if $\lambda=-\delta$ then the functional $L(\lambda)$ is not continuous. Indeed, for instance a sequence of translated Heaviside functions $H_{n}$ consisting of unitary steps at $x=n$, converges to zero in $\widetilde{M}$ :

$$
\left\|H_{n}\right\|_{\widetilde{M}}=\int_{n}^{\infty} e^{-x} d x=e^{-n}
$$

but $L(-\delta) H_{n}=\int_{\mathbb{R}^{+}} B(x) d H_{n}(x)=B(n)$, which tends to $R>0$.

(2) If suffices to show the continuity of $(\lambda, F) \mapsto L(\lambda) F$. Let $(\lambda, F) \in$ $(-\delta, \infty) \times M$ and let $\left(\lambda_{n}, F_{n}\right)$ a sequence tending to the former. Now let $\epsilon>0$, we have that

$$
\left|L\left(\lambda_{n}\right) F_{n}-L(\lambda) F\right| \leq\left|L\left(\lambda_{n}\right) F_{n}-L(\lambda) F_{n}\right|+\left|L(\lambda) F_{n}-L(\lambda) F\right| .
$$


The second term of the right hand side is less than $\frac{\epsilon}{2}$ for $n$ large enough by part 1 . The first term can be bounded as follows

$$
\begin{array}{rlrl}
\left.\mid L\left(\lambda_{n}\right) F_{n}-L(\lambda) F_{n}\right) \mid & = & & \left|\int_{\mathbb{R}^{+}} B(\tau)\left(e^{-\left(\lambda_{n}+\delta\right) \tau}-e^{-(\lambda+\delta) \tau}\right) d F_{n}(\tau)\right| \\
& \leq & R \int_{\mathbb{R}^{+}}\left|e^{-\left(\lambda_{n}+\delta\right) \tau}-e^{-(\lambda+\delta) \tau}\right| d F_{n}(\tau) \\
& \leq & c\left|\lambda_{n}-\lambda\right|<\frac{\epsilon}{2}
\end{array}
$$

for a suitable positive constant $c$ whenever $n$ is sufficiently large. Here, we have used the mean value theorem:

$$
\left|e^{-x \tau}-e^{-x^{\prime} \tau}\right| \leq \tau e^{-\tau \min \left(x, x^{\prime}\right)}\left|x-x^{\prime}\right| \leq \frac{1}{e \min \left(x, x^{\prime}\right)}\left|x-x^{\prime}\right|,
$$

and the fact that $\inf _{n}\left(\lambda_{n}+\delta\right)>0$.

Now we are concerned with the continuity of the functional $\lambda_{F}$ defined on a compact subset. Let us start by choosing a $\tilde{\delta}<\delta$ sufficiently close to $\delta$ in order that $G(-\tilde{\delta}, F) \leq 0$ for some $F \in M$. Notice that the latter is possible under assumption (11).

Let us define the set

$$
M_{K}:=\{F \in M: G(-\tilde{\delta}, F) \leq 0\} .
$$

This set is obviously non-empty and closed by Prop. 4.2 Part (2), and hence compact using Prop. 4.1.

Lemma 4.2. Under the condition $m-\delta<k S(R-1)$, the range of the functional $F \mapsto \lambda_{F}$ restricted to the compact set $M_{K}$ is contained in the interval

$$
[-\tilde{\delta}, k S(R-1)-m] \text {. }
$$

Proof. For all $F \in M_{K}$, we have that $\lambda_{F} \geq-\tilde{\delta}$. On the other hand, $G(k S(R-1)-m, F)=k S(R-L(\lambda) F)>0=G\left(\lambda_{F}, F\right)$. Hence $\lambda_{F} \leq k S(R-1)-m$ since the function $\lambda \mapsto G(\lambda, F)$ is increasing.

Proposition 4.3. Under the condition $m-\delta<k S(R-1)$, the following holds:

(1) The functional $F \mapsto \lambda_{F}$ is continuous on the compact set $M_{K}$.

(2) The functional $F \mapsto \lambda_{F}$ restricted to $M_{K}$ has an absolute maximum point $\widehat{F}$.

Proof. Let $\left\{F_{n}\right\} \in M_{K}$ be a sequence with limit $F \in M_{K}$.

Let us consider $\lambda_{n}:=\lambda_{F_{n}} \geq-\tilde{\delta}$ fulfilling $G\left(\lambda_{n}, F_{n}\right)=0$. By Lemma 4.2 there exist convergent subsequences $\left\{\lambda_{n_{k}}\right\}$. It suffices to see that their limit $\tilde{\lambda} \geq-\tilde{\delta}$ is equal to $\lambda_{F}$. Since $G(\lambda, F)$ is continuous at $(\tilde{\lambda}, F)$ by Prop. 4.2 then $0=G\left(\lambda_{n_{k}}, F_{n_{k}}\right) \rightarrow G(\tilde{\lambda}, F)=0$, and hence $G(\tilde{\lambda}, F)=0$. Therefore $\tilde{\lambda}=\lambda_{F}$.

Concerning with the maximum of the previous proposition, we have the following 
Proposition 4.4. Let $\widehat{F}$ be an absolute maximum point of the continuous functional $F \mapsto \lambda_{F}$ restricted to $M_{K}$. Then $\widehat{F}$ is also an absolute maximum point of the continuous functional $F \mapsto L\left(\lambda_{\widehat{F}}\right) F$ defined on $M$.

Proof. Let first assume that $F \in M_{K}$. Since $\widehat{F}$ is an absolute maximum point of $\lambda_{F}, \lambda_{F} \leq \lambda_{\widehat{F}}$ and since $G(\lambda, F)$ is an increasing function of $\lambda$, one has that $G\left(\lambda_{\widehat{F}}, \widehat{F}\right)=0=G\left(\lambda_{F}, F\right) \leq G\left(\lambda_{\widehat{F}}, F\right)$, and therefore

$$
0 \leq G\left(\lambda_{\widehat{F}}, F\right)-G\left(\lambda_{\widehat{F}}, \widehat{F}\right)=k S\left(-L\left(\lambda_{\widehat{F}}\right) F+L\left(\lambda_{\widehat{F}}\right) \widehat{F}\right) .
$$

On the other hand, if $F \in M \backslash M_{K}$ then $G\left(\lambda_{\widehat{F}}, \widehat{F}\right)=0<G(-\tilde{\delta}, F) \leq G\left(\lambda_{\widehat{F}}, F\right)$, using that $\lambda_{\widehat{F}} \geq-\tilde{\delta}$. Consequently, $L\left(\lambda_{\widehat{F}}\right) \widehat{F} \geq L\left(\lambda_{\widehat{F}}\right) F$ in both cases.

Before stating the main result of the section we still need the following

Lemma 4.3. Let $\psi: \mathbb{R}^{+} \rightarrow \mathbb{R}^{+}$be a continuous function with a unique absolute maximum point $\widehat{x}$. Then the functional on $M$ defined by $T(F):=\int_{[0, \infty)} \psi d F$ has a unique absolute maximum point $H_{\widehat{x}}(x):=H(x-\widehat{x})$ where $H$ is the Heaviside function.

Proof. If $F=p H_{\widehat{x}}$ with $p \in[0,1)$ then obviously $T(F)<T\left(H_{\widehat{x}}\right)$. On the other hand, if $F \in M$ is different from $p H_{\widehat{x}}$ for any $p$ then there exists a closed interval $I$ with $\mu(I)>0$ (where $\mu$ is the real measure associated to $F$ ) such that $\widehat{x} \notin I$ and

$$
\begin{aligned}
T(F) & =\int_{[0, \infty)} \psi d F=\int_{I^{c}} \psi d F+\int_{I} \psi d F \\
& <\psi(\widehat{x}) \mu\left(I^{c}\right)+\psi(\widehat{x}) \mu(I)=\psi(\widehat{x})=T\left(H_{\widehat{x}}\right)
\end{aligned}
$$

inequality follows from the fact that $\max _{I} \psi<\psi(\widehat{x})$.

Next theorem assures the existence and uniqueness of the optimal latent period in a bacteriophage infection where the lysis timing may be variable. Moreover, we are going to show that the optimal latent period is decreasing with respect to both the number of susceptible bacteria (under a suitable bacterial mortality) and the quality of bacteria quantified by the parameter $R$ as in [18].

Let us recall the assumptions on the burst size given in Section 2. $B(\tau)$ is a bounded and continuous function, $B(\tau) \equiv 0$ for $\tau \leq E$, strictly increasing with $B^{\prime \prime}(\tau)<0$ for $\tau>E$ and the maximum burst size $\lim _{\tau \rightarrow l} B(\tau)=R$. Let us rewrite the burst size as $B(\tau)=R b(\tau)$ so that the function $b(\tau)$ represents a normalized burst size with $\lim _{\tau \rightarrow l} b(\tau)=1$. Now we are ready to state the main result of the section.

Theorem 4.1 (optimal latent period). Under condition $m-\delta<k S(R-1)$, there exists a unique probability distribution function $\widehat{F}$ such that the growth bound of the solution semigroup of (2) $\lambda_{F}$ is maximal. Moreover, 
(1) $\widehat{F}$ has the form $\widehat{F}(\tau)=\mathcal{X}_{\widehat{l}, \infty)}(\tau)$ where $\widehat{l}>E$ is the unique solution of the non-linear equation

$$
\frac{b^{\prime}(l)}{b(l)}+m-\delta=k S\left(R b(l) e^{-\frac{b^{\prime}(l)}{b(l)} l}-1\right),
$$

that is, the optimal latent period is $\widehat{l}$ with probability 1 .

(2) The optimal latent period $\widehat{l}$ is strictly decreasing with respect to $R$. On the other hand, there exists a critical value $\delta_{c}>m$ of the bacterial mortality such that $\widehat{l}$ is strictly decreasing with respect to the number of susceptible bacteria $S$ if and only if $\delta<\delta_{c}$.

Proof. Let condition $m-\delta<k S(R-1)$ hold.

If $F \in M_{1} \backslash M_{K}$ then either $\lambda_{F}$ does not exist and the growth bound of the solution semigroup is less than or equal to $-\delta$, or $G(-\tilde{\delta}, F)>0$ and $\lambda_{F}<-\tilde{\delta}$. Therefore we can restrict the optimization of the growth bound to $F \in M_{K}$.

By Prop. 4.3 the functional $F \mapsto \lambda_{F}$ restricted to $M_{K}$ has an absolute maximum point $\widehat{F}$ with value $\widehat{\lambda}:=\lambda_{\widehat{F}}$, which by Prop. 4.4 is also an absolute maximum point of the functional $F \mapsto L(\widehat{\lambda}) F$ defined on $M$. According to the definition in $(12)$,

$$
L(\widehat{\lambda}) F=\int_{\mathbb{R}^{+}} \psi(\tau) d F(\tau)
$$

with

$$
\psi(\tau):=R b(\tau) e^{-(\widehat{\lambda}+\delta) \tau} .
$$

The continuous function $\psi: \mathbb{R}^{+} \rightarrow \mathbb{R}^{+}$defined above has a unique absolute maximum point $\widehat{l}>E$. Indeed,

$$
\psi^{\prime}(\tau)=\operatorname{Re}^{-(\widehat{\lambda}+\delta) \tau}\left(b^{\prime}(\tau)-(\widehat{\lambda}+\delta) b(\tau)\right)
$$

and thus we have that the critical points are the solutions of

$$
\widehat{\lambda}=\frac{b^{\prime}(\tau)}{b(\tau)}-\delta, \quad \tau>E .
$$

Finally, there exists a unique critical point $\widehat{l}>E$ of $\psi(\tau)$ since according to the assumptions on the burst size, the function $\frac{b^{\prime}(\tau)}{b(\tau)}, \tau>E$, in (15) is strictly decreasing and takes all the positive values. Moreover, the critical point $\widehat{l}>E$ is a maximum point since $\psi^{\prime \prime}(\widehat{l})<0$.

Now, by Lemma 4.3 with $\psi$ defined by (14), the functional $F \mapsto L(\widehat{\lambda}) F$ has a unique absolute maximum point $\mathcal{X}_{\widehat{[}, \infty)}$, with $\widehat{l}>E$ being the solution of (15). Therefore $\widehat{F}$, which is an absolute maximum point given by Prop. 4.3 , must be $\mathcal{X}_{\widehat{l}, \infty)}$. Furthermore, this probability distribution function is the unique absolute maximum point of the functional $\lambda_{F}$ since if there exists another one $\bar{F}$ then 
it will be also an absolute maximum point of the functional $F \mapsto L\left(\lambda_{\bar{F}}\right) F$ but $\lambda_{\bar{F}}=\lambda_{\widehat{F}}=\widehat{\lambda}$ and consequently $\bar{F}=\mathcal{X}_{\widehat{[l}, \infty)}$.

To end up, we have that

(1) Combining the characteristic equation (9) at $F=\mathcal{X}_{\widehat{[}, \infty}$, that is $G\left(\widehat{\lambda}, \mathcal{X}_{\widehat{[}, \infty}\right)$ $=0$, with the condition of critical point $\widehat{\lambda}=\frac{b^{\prime}(\widehat{l})}{b(\widehat{l})}-\delta$, we get that $\widehat{l}>E$ is a solution of the scalar non-linear equation (13). Actually, it is the unique solution according to the type of monotonicity assumed on the burst size.

(2) To prove the second part of the claim we have to compute the sign of two derivatives. Indeed, the optimal latent period $\widehat{l}>E$ is strictly decreasing with respect to $R$ since

$$
\frac{d \widehat{l}}{d R}=\frac{-k S b(\widehat{l}) e^{-\frac{b^{\prime}(\widehat{l})}{b(\hat{l})}}}{k S R \widehat{l} e^{-\frac{b^{\prime}(\widehat{l})}{b(\hat{l})}} \widehat{l} \frac{b^{2}(\widehat{l})-b(\widehat{l}) b^{\prime \prime}(\widehat{l})}{b(\widehat{l})}+\frac{b^{\prime 2}(\widehat{l})-b(\widehat{l}) b^{\prime \prime}(\widehat{l})}{b^{2}(\widehat{l})}}
$$

is negative according to the type of monotonicity that we have assumed on the normalized burst size, i.e. $b^{\prime \prime}(\tau)<0$ for $\tau>E$. The expression in (16) follows from the equation (13) as an implicit derivative.

On the other hand, the fact that the optimal latent period $\widehat{l}>E$ is strictly decreasing with respect to $S$ is derived as follows. Implicitly differentiating in equation (13), we have that

$$
\frac{d \widehat{l}}{d S}=\frac{k\left(1-R b(\widehat{l}) e^{-\frac{b^{\prime}(\widehat{l})}{b(\hat{l})}}\right)}{k S R \widehat{l} e^{-\frac{b^{\prime}(\widehat{l})}{b(\hat{l})}} \frac{b^{\prime 2}(\widehat{l})-b(\widehat{l}) b^{\prime \prime}(\widehat{l})}{b(\widehat{l})}+\frac{b^{\prime 2}(\widehat{l})-b(\widehat{l}) b^{\prime \prime}(\widehat{l})}{b^{2}(\widehat{l})}},
$$

which is negative if and only if

$$
R b(\widehat{l}) e^{-\frac{b^{\prime}(\widehat{l})}{b(\hat{l})}}>1 .
$$

Now, one has that $\widehat{l}>E$ which is the unique solution of (13), is also the unique root of the right hand side of (13), i.e. $R b(l) e^{-\frac{b^{\prime}(l)}{b(l)} \widehat{l}}=1$, if and only $\delta=\delta_{c}:=m+\frac{b^{\prime}(\widehat{l})}{b(\widehat{l})}>m$ (a critical value of the bacterial mortality). Moreover, condition (18) holds if and only if $\delta<\delta_{c}$. These results follow from an analysis of the left and right hand sides of (13) as functions of $l>E$.*

According to (15) at $\tau=\widehat{l}$, once we know the optimal latent period $\widehat{l}$ as the solution of equation (13), the maximal fitness (the maximal growth bound of 
the solution semigroup) is given by

$$
\widehat{\lambda}=\frac{b^{\prime}(\widehat{l})}{b(\widehat{l})}-\delta=\frac{B^{\prime}(\widehat{l})}{B(\widehat{l})}-\delta>-\delta .
$$

\section{Appendix A. Existence And uniqueness of solutions}

In this appendix we are going to show the existence and uniqueness of solutions of the model described by system (2), or equivalently by (3) as a single equation. Let us consider an initial condition $\left(v_{0}(\cdot), P_{0}\right) \in X:=L^{1}(0, l) \times \mathbb{R}$ and a fixed interval $\left[0, t_{f}\right]$ with $t_{f}<l$. Now equation (3) for $P(t), 0 \leq t \leq t_{f}$, reads as

$$
\begin{aligned}
P(t)=P_{0} e^{-\nu t} & +\int_{0}^{t}\left(k S \int_{(0, s]} B(\tau) P(s-\tau) e^{-\delta \tau} d F(\tau)\right. \\
& \left.+e^{-\delta s} \int_{(s, l]} B(\tau) v_{0}(\tau-s) \frac{d F(\tau)}{1-F(\tau-s)}\right) e^{-\nu(t-s)} d s .
\end{aligned}
$$

We recall that $\nu:=m+k S>0, \sup _{\tau>0} B(\tau)=R$, and $F(\tau)$ is a general probability distribution function. Let $\mathcal{C}:=\mathcal{C}\left(\left[0, t_{f}\right], \mathbb{R}\right)$ be the Banach space of continuous functions with the supremum norm. Let $\mathcal{B}: \mathcal{C} \rightarrow \mathcal{C}$ be the following linear operator

$$
(\mathcal{B P})(t):=k S \int_{0}^{t} e^{-\nu(t-s)} \int_{(0, s]} B(\tau) P(s-\tau) e^{-\delta \tau} d F(\tau) d s
$$

and, for $v_{0} \in L^{1}(0, l)$ and $P_{0}$ real value, let $V_{0}$ be the function

$$
V_{0}(t):=P_{0} e^{-\nu t}+\int_{0}^{t} e^{-\nu(t-s)-\delta s} \int_{(s, l]} B(\tau) v_{0}(\tau-s) \frac{d F(\tau)}{1-F(\tau-s)} d s .
$$

Thus, equation (19) is written in short as

$$
P(t)=(\mathcal{B P})(t)+V_{0}(t) .
$$

Proposition A.1. The operator $\mathcal{B}$ is well-defined and $V_{0}(\cdot) \in \mathcal{C}$.

Proof. Firstly, we have to show that if $P(\cdot) \in \mathcal{C}$ then $(\mathcal{B P})(\cdot) \in \mathcal{C}$. Indeed, let us define $L_{1}(s):=k S \int_{(0, s]} B(\tau) P(s-\tau) e^{-\delta \tau} d F(\tau)$, then $(\mathcal{B} P)(t)=\int_{0}^{t} e^{-\nu(t-s)} L_{1}(s) d s$. It suffices to show that $L_{1}(\cdot) \in L^{1}\left(0, t_{f}\right)$ :

$$
\begin{aligned}
\int_{0}^{t_{f}}\left|L_{1}(s)\right| d s & \leq k S \int_{0}^{t_{f}} \int_{(0, s]} B(\tau)|P(s-\tau)| e^{-\delta \tau} d F(\tau) d s \\
& \leq k S R \int_{\left(0, t_{f}\right]} \int_{\tau}^{t_{f}}|P(s-\tau)| d s d F(\tau) \\
& =k S R \int_{\left(0, t_{f}\right]} \int_{0}^{t_{f}-\tau}|P(\sigma)| d \sigma d F(\tau) \leq k S R\|P\|_{L^{1}\left(0, t_{f}\right)} .
\end{aligned}
$$

On the other hand, let us show the continuity of $V_{0}$ by a similar argument. Making the linear change of variables $\tau-s=\sigma, \tau=\tau$ to the double integral 
$\int_{0}^{t_{f}} \int_{(s, l]} v_{0}(\tau-s) \frac{d F(\tau)}{1-F(\tau-s)} d s$, the region of integration is transformed into $\{(\tau, \sigma) \in$ $\left.[0, l]^{2}: \tau-t_{f}<\sigma<\tau\right\}$ and therefore

$$
\begin{aligned}
\int_{0}^{t_{f}} \int_{(s, l]} v_{0}(\tau-s) \frac{d F(\tau)}{1-F(\tau-s)} d s \\
\quad=\left(\int_{0}^{l-t_{f}} \int_{\sigma}^{\sigma+t_{f}}+\int_{l-t_{f}}^{l} \int_{\sigma}^{l}\right) \frac{v_{0}(\sigma)}{1-F(\sigma)} d F(\tau) d \sigma \\
\quad=\int_{0}^{l-t_{f}} \frac{F\left(\sigma+t_{f}\right)-F(\sigma)}{1-F(\sigma)} v_{0}(\sigma) d \sigma+\int_{l-t_{f}}^{l} \frac{1-F(\sigma)}{1-F(\sigma)} v_{0}(\sigma) d \sigma \\
\leq\left\|v_{0}\right\|_{L^{1}(0, l)} .
\end{aligned}
$$

Thus it follows that the function $L_{0}(s):=e^{-\delta s} \int_{(s, l]} B(\tau) v_{0}(\tau-s) \frac{d F(\tau)}{1-F(\tau-s)}$ belongs to $L^{1}\left(0, t_{f}\right)$ with

$$
\left\|L_{0}\right\|_{L^{1}\left(0, t_{f}\right)} \leq R\left\|v_{0}\right\|_{L^{1}(0, l)} .
$$

Then $V_{0}(\cdot) \in \mathcal{C}$, using the same argument as before, with norm

$$
\left\|V_{0}\right\|_{\mathcal{C}} \leq \max (1, R)\left\|\left(v_{0}, P_{0}\right)\right\|_{L^{1}(0, l) \times \mathbb{R}} \cdot *
$$

Theorem A.1. For any initial condition $\left(v_{0}(\cdot), P_{0}\right) \in X$, system (2) has a unique local solution. Moreover, if the initial condition is non-negative then the solution is non-negative.

Proof. Firstly, let us show the existence and uniqueness of solution of equation $(20)$. We can compute $P(t)$ from $(20)$ if the operator $(I d-\mathcal{B})$ is invertible since $P(t)=\left((I d-\mathcal{B})^{-1} V_{0}\right)(t)$. If $\|\mathcal{B}\|<1$ then the linear operator $(I d-\mathcal{B})$ is invertible.

$$
\begin{aligned}
\|\mathcal{B}\| & =\sup _{\|\phi\|_{\mathcal{C}} \leq 1}\|(\mathcal{B} \phi)(t)\|_{\mathcal{C}} \\
& =\sup _{\|\phi\|_{\mathcal{C}} \leq 1} \sup _{t \in\left[0, t_{f}\right]}\left|k S \int_{0}^{t} e^{-\nu(t-s)} \int_{(0, s]} B(\tau) \phi(s-\tau) e^{-\delta \tau} d F(\tau) d s\right| \\
& \leq k S R \sup _{\|\phi\|_{\mathcal{C}} \leq 1} \sup _{t \in\left[0, t_{f}\right]} \int_{0}^{t} \int_{(0, s]}|\phi(s-\tau)| d F(\tau) d s \\
& \leq k S R \sup _{\|\phi\|_{\mathcal{C}} \leq 1}\|\phi\|_{\mathcal{C}} t_{f}=k S R t_{f} .
\end{aligned}
$$

So, taking $t_{f}<\frac{1}{k S R}$ then we can assure that $\|\mathcal{B}\|<1$. In this fixed period of time $\left[0, t_{f}\right]$, the solution can be written as $P(t)=\sum_{n \geq 0}\left(\mathcal{B}^{n} V_{0}\right)(t)$ showing that the solution is non-negative if it is the initial condition, i.e. $\left(v_{0}(\cdot), P_{0}\right) \in$ $L_{+}^{1}(0, l) \times \mathbb{R}_{+}$. Once we know the solution of $(20)$, the solution of system $(2)$ is readily recovered. 
Let us remark that for the usual initial conditions used in laboratory experiments, i.e. $v_{0}(\tau) \equiv 0$ and $P_{0}>0$, then $V_{0}(t)=P_{0} e^{-\nu t}$ and the solution of $(2)$ is given by $P(t)=P_{0}(I d-\mathcal{B})^{-1} \exp (-\nu t)>0$, and $v(\tau, t)=$ $k S P_{0}(1-F(\tau)) e^{-\delta \tau}(I d-\mathcal{B})^{-1} \exp (-\nu(t-\tau)), \tau<t$, and zero otherwise.

A standard (and tedious) computation shows that the solution of system (2) defines a family of bounded linear operators in $X$,

$$
\mathcal{S}(t)\left(\begin{array}{c}
v_{0}(\cdot) \\
P_{0}
\end{array}\right):=\left(\begin{array}{c}
v(\cdot, t) \\
P(t)
\end{array}\right)
$$

which fulfills the (local) semigroup condition. Next let us show that the strong continuity condition is also satisfied. We have

Proposition A.2. The solution semigroup of system (2) yields a strongly continuous (local) semigroup of bounded linear operators in $X$.

Proof. We have to show that

$$
\lim _{t \rightarrow 0^{+}} \mathcal{S}(t)\left(\begin{array}{c}
v_{0}(\cdot) \\
P_{0}
\end{array}\right)=\left(\begin{array}{c}
v_{0}(\cdot) \\
P_{0}
\end{array}\right) \text { in } X .
$$

The second component of the $\operatorname{limit}_{\text {, }} \lim _{t \rightarrow 0^{+}} P(t)=P_{0}$, is trivial using Prop. A.1. On the other hand, the first component, i.e. $\lim _{t \rightarrow 0^{+}}\left\|v(\cdot, t)-v_{0}(\cdot)\right\|_{L^{1}(0, l)}=0$, is derived as follows. From (2) and $t<l$

$$
\begin{aligned}
\int_{0}^{t} \mid k S P(t-\tau)(1-F(\tau)) e^{-\delta \tau} & -v_{0}(\tau) \mid d \tau \\
& +\int_{t}^{l}\left|v_{0}(\tau-t) \frac{1-F(\tau)}{1-F(\tau-t)} e^{-\delta t}-v_{0}(\tau)\right| d \tau .
\end{aligned}
$$

The first integral tends to 0 , as time $t$ tends to $0^{+}$, since $P$ and $v_{0}$ are integrable functions. Regarding the second one,

$$
\begin{aligned}
\int_{t}^{l} \mid & v_{0}(\tau-t) \frac{1-F(\tau)}{1-F(\tau-t)} e^{-\delta t}-v_{0}(\tau) \mid d \tau \\
\quad & \int_{0}^{l-t}\left|v_{0}(s) \frac{1-F(t+s)}{1-F(s)} e^{-\delta t}-v_{0}(s+t)\right| d s \\
& \leq \int_{0}^{l-t}\left|v_{0}(s) \frac{1-F(t+s)}{1-F(s)} e^{-\delta t}-v_{0}(s)\right| d s+\int_{0}^{l-t}\left|v_{0}(s+t)-v_{0}(s)\right| d s .
\end{aligned}
$$

Now, since $\left(v_{0}(s) \frac{1-F(t+s)}{1-F(s)} e^{-\delta t}-v_{0}(s)\right) \mathcal{X}_{[0, l-t]}(s)$ tends pointwise to 0 as time $t$ tends to $0^{+}$if $s \neq l$ is a continuity point of $F$, and its absolute value is bounded above by $\left|v_{0}(s)\right|$, then by the dominated convergence theorem we can conclude that the convergence is in the $L^{1}(0, l)$ sense. Finally, the second integral above also tends to 0 , as $t$ tends to $0^{+}$, by a well known property of the integral. 
Finally, we have the existence and uniqueness of global solutions which follows as a corollary of the previous statements. Indeed, using the uniqueness of solutions, the semigroup property and the fact that the existence time $t_{f}>0$ does not depend on the initial condition, we have the following

Theorem A.2. The local solution of system (2) given by Theorem A.1 is actually a global solution and defines a strongly continuous linear semigroup.

\section{ACKNOWLEDGMENTS}

The author JR wishes to thank the Centre de Recerca Matemàtica for the hospitality he enjoyed within the research program on "Mathematical Biology: Modelling and Differential Equations" in March 2009. He is specially grateful to the CRM staff for the attention received during the stay.

\section{REFERENCES}

[1] S.T. Abedon, Selection for Bacteriophage Latent Period Length by Bacterial Density: A Theoretical Examination, Microbial Ecology 18, (1989) 79-88.

[2] S.T. Abedon, T.D. Herschler, D. Stopar, Bacteriophage Latent-Period Evolution as a Response to Resource Availability, Applied and Enviromental Microbiology 67 (2001), 4233-4241.

[3] C.D. Aliprantis, K.C. Border, Infinite Dimensional Analysis: A Hitchhiker's Guide, Springer-Verlag, Berlin, 1999.

[4] W. Arendt, A. Grabosch, G. Greiner, U. groh, H.P. Lotz, U. Moustakas, R. Nagel, F. Neubrander and U. Schlotterbeck, One-Parameter Semigroups of Positive Operators, Springer-Verlag, Berlin, 1986.

[5] E. Beretta and Y. Kuang, Modeling and analysis of a marine bacteriophage infection, Math. Biosci. 149 (1998), 57-76.

[6] E. Beretta and Y. Kuang, Modeling and analysis of a marine bacteriophage infection with latency period, Nonlinear Anal. Real World Appl. 2 (2001), 35-74.

[7] A. Calsina and J. Ripoll, A general structured model for a sequential hermaphrodite population, Math. Biosci. 208 (2007), 393-418.

[8] A. Calsina and J. Ripoll, Evolution of age-dependent sex-reversal under adaptive dynamics, J. Math. Biol. (2009).

[9] Ph. Clément, H. J. A. M. Heijmans, S. Angenent, C. J. van Duijn, B. de Pagter Oneparameter semigroups, CWI Monographs 5, North-Holland Publishing Co., Amsterdam, 1987.

[10] S.A. Gourley and Y. Kuang, A delay reaction-diffusion model of the spread of bacteriophage infection, SIAM J. Appl. Math. 65 (2004/05), 550-566.

[11] H. Hadas, M. Einav, I. Fishov and A. Zaritsky, Bacteriophage T4 development depends on the physiology of its host Escherichia coli, Microbiology 143 (1997) 179-185.

[12] M.G. Krein, D. Milman, On extreme points of regular convex sets, Studia Mathematica 9 (1940), 133-138.

[13] D. Nualart, M. Sanz, Curs de probabilitats, Estadística i análisis de datos 5, PPU, Barcelona, 1990.

[14] A. Rabinovitch, H. Hadas, M. Einav, Z. Melamed and A. Zaritski, Model for bacteriophage T4 development in Escherichia coli, J. Bacteriol. 181 (1999), 1677-1683. 
[15] J. Ripoll, Evolution of sex-ratio in structured population dynamics, Doctoral Thesis, Universitat de Barcelona, 2005.

[16] W. Rudin Real and complex analysis (3rd edition), McGraw-Hill, New York, 1987.

[17] I.-N. Wang, Lysis Timing and Bacteriophage Fitness, Genetics Society of America 172 (2006), 17-26.

[18] I.-N. Wang, D.E. Dykhuizen and I.B. Slobodkin, The evolution of phage lysis timing, Evol. Ecol. 10 (1996), 545-558.

Àngel Calsina

Departament de Matemàtiques

Universitat Autònoma de BARCELONA

08193 Bellaterra, Barcelona, Spain

E-mail address: acalsina@mat.uab.cat

Josep M. Palmada

Departament d'Informàtica i Matemàtica Aplicada

Universitat DE Girona

Campus Montilivi, 17071 Girona, Spain

E-mail address: jpalmada@ima.udg.es

JoRDi RIPOLL

Departament D'Informàtica I Matemàtica Aplicada

Universitat DE Girona

Campus Montilivi, 17071 Girona, Spain

E-mail address: jripoll@ima.udg.es 